\title{
Pattern of pathogens from surgical wound infections in a Nigerian hospital and their
}

antimicrobial susceptibility profiles

Ezekiel Olugbenga Akinkunmi ${ }^{1}$, Abdul-Rashid Adesunkanmi ${ }^{2}$, Adebayo Lamikanra ${ }^{1}$

1. Obafemi Awolowo University, Ile-Ife, Department of Pharmaceutic

2. Obafemi Awolowo University, Ile-Ife, Department of Surgery

\section{Abstract:}

Background: In surgical patients, infection is an important cause of morbidity and mortality. A prospective study to find the pattern of microorganisms responsible for post operative wound infections and their antibiotic susceptibility profile was therefore conducted.

Setting and Methods: Surgical wards in Obafemi Awolowo University Teaching Hospital Complex, Ile-Ife, Nigeria. Isolation, identification and antimicrobial susceptibility screening of organisms were done employing standard microbiological techniques.

Results: Bacterial pathogens were isolated from all the specimens while the yeast Candida species (spp) was isolated from $12.4 \%$. Staphylococcus aureus was the most frequent organism isolated accounting for $23(18.3 \%)$ of a total of 126 isolates Other organisms were Pseudomonas aeruginosa and Bacillus spp 11.1\% each; Escherichia coli 10.3\%; Candida spp $8.7 \%$ Coagulase negative staphylococci 8.7\% : Pseudomonas spp 6.3\%; Serratia odorifera 4.7\%; Bacteroides 4.0\%; Enterococus ( $3.2 \%$ the remining 作 eral, resistance to the $\beta$-lactam antibiotics was above $98 \%$, whilst more than $70 \%$ of isolates were resistant to erythromycin,
fusidic acid and tobramycin. fusidic acid and tobramycin

Conclusions:The infections were polymicrobic and multidrug resistant. The quinolones, ciprofloxacin and ofloxacin, should be used as frontline drugs in the management of surgical wound infections at the hospital.

Keywords: surgical wound infections, susceptibility, bacterial pathogens, antibiotics

DOI: http://dx.doi.org/10.4314/ahs.v14i4.5

\section{Introduction}

In spite of the progress in surgery, surgical techniques and antibiotic prophylaxis ${ }^{1,2,3}$, postoperative infections remain the commonest postoperative complications and one of the most frequently encountered nosocomial infections worldwide ${ }^{4,5}$. The incidence of these infections has been estimated to be $15.45 \%$ and $11.32 \%$ by the Center for Disease Control and Prevention (CDC) USA and the UK Nosocomial Infection Surveillance respectively ${ }^{6}$. These infections lead to increase morbidity with the attendant increase in cost of therapy ${ }^{7}$. The high incidence and prevalence of postoperative wound infections also result in increasing demand on the lim-

\begin{tabular}{|l|}
\hline Corresponding author: \\
Ezekiel Olugbenga Akinkunmi \\
Department of Pharmaceutics, \\
Obafemi Awolowo University, Ile-Ife, Nigeria. \\
Email: eoakinmi@oauife.edu.ng, \\
akinkeroo@yahoo.com \\
Tel: +2348036548975 \\
\hline
\end{tabular}

ited resources available to healthcare delivery eventually resulting in high degree of mortality ${ }^{1,7}$. As a result of these problems, routine surveillance for hospital acquired wound infections, including surgical wound infections, is recommended by both the CDC and the Surgical Infection Society in USA (SIS) ${ }^{2,7}$.

Risk of wound infection varies with the type of surgery and surgical operations have been classified into clean, clean-contaminated, contaminated and dirty ${ }^{8,9}$. A clean wound is an incision through un-inflamed tissue in which the wound is primarily closed. In this wound type only closed drainage systems are used and there is no breach in aseptic technique and the viscus is no opened. A clean-contaminated wound is one (that is otherwise clean) created at emergency surgery and in which the un-inflamed upper gastrointestinal tract, normal gall bladder and urinary bladder are opened but there is no spillage of contents and there is mino break in aseptic technique. Contaminated wounds are traumatic wounds less than 6 hours old and wounds in which the inflamed upper gastrointestinal tract and obstructed urinary bladder are opened or spillage of contents occurs. In these wounds there are major breaks in sterile technique. Dirty wounds are associated with presence of pus and may include intra-peritoneal abscess formation or visceral perforation and traumatic wounds more than 6 hours old ${ }^{8,9}$.

The choice of treatment for post-surgical infections requires an understanding of the usual infectious flora, available antimicrobial agents and susceptibility patterns of the infecting organisms as these would be helpful in the selection of empiric antimicrobial therapy and also on infection control measures in the health institutions ${ }^{10,11}$. The investigation of the microbiologic spectrum and antibiotic susceptibility of isolates in surgical would infections is therefore of increasing importance bearing in mind the increasing antibiotic resistance by microorganisms and the high incidence of surgical infections caused by these resistant organisms ${ }^{11}$.

Anaerobic bacteriology is expensive and requires special facilities and expertise to perform. It is not readily available in many hospitals in the developing countries. Therefore most studies from developing countries do not incorporate anaerobic bacteriology in the study of surgical wound infection despite the reported significant roles that anaerobes play in such infections ${ }^{1}$.

In this study we report on the microbiological spectrum of post operative wound infections in a Nigerian Teaching Hospital and the antimicrobial susceptibility profiles with a view to providing guideline to the clinicians for making rational decision over the choice of antibiotic in the management of surgical site infection.

\section{Materials and Methods}

\section{Study centre}

The study was conducted at Obafemi Awolowo University Teaching Hospital Complex (OAUTHC), Ile-Ife Nigeria for a period of 2 years from September 2005 to Sept 2007 after appropriate approval were obtained and following standard guidelines. The hospital caters for a wide variety of patients ranging from high to low income level patients. The teaching hospital provides health care services for people from over five different states in the South Western parts of Nigeria: Oyo, Osun, Ondo, Ekiti and Kwara States. During the collection of specimens for the study, hospital activities were disrupted at several points by industrial actions undertaken by several staff unions within the hospital, hence a smaller number of surgical operations than expected were carried out in the centre.
Patients

Samples were obtained from the surgical sites of 89 hospitalised patients who showed clinical evidence of post operative wound infections as diagnosed by the physicians. In such cases, a surgical wound with pus or seropurulent discharge and with signs of sepsis was considered as surgical site infection. In all the cases, the detection of infection was within thirty days of surgery. Wounds with cellulitis and no drainage and suture abscesses were not included in the study. The patients included 56 males and $14.6 \%$ of all the patients fall below the age of 15 years. The patients were diagnosed as having cellulitis, breast cancers, typhoid perforation, biliary atresia, scalp necrosis, burns, faecal fistula and abscesses. Information about patients regarding age, sex, date of admission, associated co-morbid conditions, type of surgery, type of wounds and preoperative antibiotic prophylaxis were collected in a case record.

\section{Collection of Samples}

All consenting general surgical patients with wound infection were used for the study. Specimens were collected using standard collection techniques. ${ }^{12}$ Briefly, a sterile cotton-wool swab was used to collect a sample from the infected site. The swabs were introduced gently into the wound sites and rotating the swab tips in the wound, taking care to avoid contamination of specimen with commensals from the skin, and then immersed immediately in a MacCartney bottle containing Stuart Transport medium (Merck, Germany). Each sample bottle was labeled carefully and transported to the laboratory immediately for microbiological investigations.

\section{Isolation of organisms}

At the laboratory, the swabs were inoculated onto freshly prepared blood agar and Sabouraud Dextrose agar [SDA] (Oxoid, England) plates and incubated aerobically at $37^{\circ} \mathrm{C}$ for $24-48$ hours for the blood agar and $25^{\circ} \mathrm{C}$ for 3-5 days for SDA. Anaerobic incubation was also done by culturing on fastidious anaerobic blood agar (LAB M, England) plates prepared according to the instruction of the manufacturer and incubated anaerobically in an anaerobic jar supplied with a commercial gas generating kit (BBL Cockleysville, USA) that provided an atmosphere of $1 \% \mathrm{O} 2 / 8 \% \mathrm{CO} 2$ in accordance to the manufacturers instruction. Incubation was done at $37^{\circ} \mathrm{C}$ for 3 to 5 days. Distinct well separated colonies growing on such plates were then sub-cultured onto newly prepared blood agar plates as appropriate. Isolates were maintained by cryopreservation using the medium of Gibson and Khoury ${ }^{13}$ and in nutrient agar stabs.

African Health Sciences Vol 14 Issue 4, December 2014 
Identification of isolates

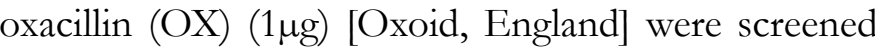
The characterization of bacterial isolates was based on The zones of inhibition were measured and interpretastandard biochemical tests ${ }^{14}$ which were performed on tion was in accordance with manufacturers' instruction the isolates and these include; gram stain, morphologi- (AB, Biodisc; PDM Interpretative chart). Staphylococcal and cultural characteristics of colonies on MacCo- cus aureus NCTC 6571 and Escherichia coli NCTC nkey agar, Eosine Methylene Blue agar, Brilliant Green 10418 were used as controls.

Agar, and Mannitol Salt Agar, haemolysis, catalase production and test for oxidase. Coagulase tests were done Results

$\begin{array}{ll}\text { for both free and bound coagulase to confirm patho- } & \text { All the specimens obtained yielded growth of bacte- } \\ \text { genic staphylococci. } & \text { ria. A total of } 126 \text { isolates were recovered from the } 89\end{array}$ genic staphylococci. ria. A total of 126 isolates were recovered from the 89
samples taken. There were $73.0 \%$ dirty surgical wounds described $^{15}$. Further tests carried out for gram negative which gave $74.6 \%$ of the isolates and $27.0 \%$ contamiisolates included motility test, nitrate reduction, hydro- nated surgical wounds which accounted for the remaingen sulphide production, indole production, Methyl ing isolates. Abdominal wounds were most frequent acRed - Voges Proskauer tests, citrate utilization, Triple counting for $44.9 \%$, followed by leg wounds, $18.0 \%$; - Sugar Iron Agar tests and sugar fermentation tests chest wall, 9.0\% and burns, 9.0\%. Correspondingly, using maltose, mannose, mannitol, glucose, sorbitol, abdominal wounds accounted for the majority of the raffinose and arabinose ${ }^{14}$.

\section{Antibiotic resistance testing}

Resistance to antibiotics was determined for the staphylococci isolates using the standard disc diffusion methods as described by the Clinical Laboratory Standard Institute (CLSI) ${ }^{16}$. The test media was Isosensitest Agar supplemented with whole blood for aerobes and chocoinclude ofloxaxin (Of), chloramphenicol (Chl), cepha-

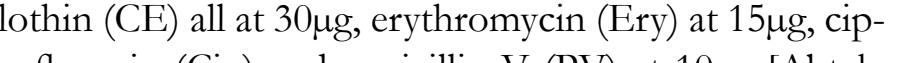
rofloxacin (Cip) and penicillin V (PV) at 10ug [Abtek,

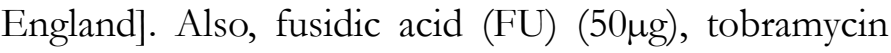
wound pathogens isolated $(39.7 \%)$ while leg wounds, burns, and chest wall wounds accounted for $17.5 \%$ $10.3 \%$ and $8.7 \%$ of the pathogens respectively.

The count of aerobic bacteria in the samples was high. Only $8.0 \%$ of the isolates were anaerobes and these anaerobes were isolated from $11.7 \%$ of the patients. Also, some of the infections were caused by the yeast Candida spp as $12.4 \%$ of the patients yielded this pathogen.

A single pathogen was identified in $56.2 \%$ patients, 2 agents were isolated from $33.7 \%$ while 3 agents were ymicrobic infections did not follow any specific pattern (Table 2). late agar for anaerobes ${ }^{22}$. The antibiotic discs employed (TM) $(30 \mu \mathrm{g})$, trimethoprim (TR) (5 $\mu \mathrm{g})$, cefadroxil (DX)

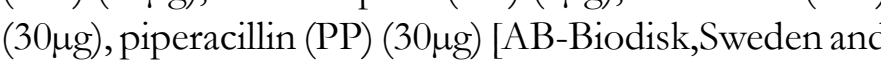

TABLE 2. The distribution of bacterial isolates in relation to type of surgical operation and type of wound that develop infection

\begin{tabular}{|c|c|c|}
\hline Wound type & $\begin{array}{l}\text { Surgical Operations } \\
\text { (no of cases) }\end{array}$ & Isolates (no of isolates) \\
\hline Head / Skull & Dirty (1) & S. aureus (1), Pseudomonas spp (1), Bacteroides (1) \\
\hline$(n=4)$ & Contaminated (3) & S. aureus (1), E. coli (1), Proteus mirabilis (1), Ps. aeruginosa (2), Candida spp (2) \\
\hline Chest wall $(\mathrm{n}=8)$ & Dirty (8) & $\begin{array}{l}\text { S. aureus (1), E. coli (1), Enterobacter agglomerans (1), Proteus penneri (1), Pseudomonas aeruginosa (2), } \\
\text { Pseudomonas spp (3), Anaerobic cocci (1), Bacteroides (1) }\end{array}$ \\
\hline Upper $\operatorname{arm}(\mathrm{n}=1)$ & Contaminated (1) & P. aeruginosa (1), Pseudomonas spp (1), Candida spp (1) \\
\hline Facial $(\mathrm{n}=1)$ & Contaminated (1) & Staphylococcus spp (1) \\
\hline $\begin{array}{l}\text { Trunk/Traumatic } \\
(\mathrm{n}=1)\end{array}$ & Contaminated (1) & P. aeruginosa (1), Candida spp (1) \\
\hline Abdomen $(\mathrm{n}=40)$ & Dirty (30) & $\begin{array}{l}\text { S. aureus (8), S. epidermidis (1), S. saprophyticus (1), S. xylosus (1), Staphylococcus spp (2), Bacillus spp (7), } \\
\text { Enterococcus spp (3), E. coli (5), S. odorifera (1), P. aeruginosa (3), Ps. maltophila (1), Pseudomonas spp (1), Anaerobic } \\
\text { cocci (2), Gram positive anaerobic rods (1), Candida spp (1) }\end{array}$ \\
\hline & Contaminated (10) & S. aureus (4), S. epidermidis (1), Bacillus spp (2), E. coli (3), K. pneumonia (1), S. odorifera (1) \\
\hline Buttock $(n=3)$ & Dirty (3) & S. aureus (1), S. epidermidis (1), E. agglomerans (1), Candida spp (2) \\
\hline Scrotal $(\mathrm{n}=4)$ & Dirty (4) & S. aureus (1), S. epidermidis (1), S. saprophyticus (1), K. pneumonia (1) \\
\hline $\begin{array}{l}\mathrm{Leg} / \mathrm{Limb} \\
(\mathrm{n}=16)\end{array}$ & Dirty (16) & $\begin{array}{l}\text { S. aureus (3), S. epidermidis (1), Bacillus spp (2), E. coli (2), P. mirabilis (1), Citrobacter spp (1), Proteus spp (2), S. } \\
\text { odorifera (3), P. arruginosa (2), Anaerobic cocci (1), Bacteroides (2), Candida spp (3) }\end{array}$ \\
\hline Burns $(\mathrm{n}=8)$ & Contaminated (8) & $\begin{array}{l}\text { S. aureus (3), Bacillus spp (1), Enterococcusspp (1), E. coli (1), E. agglomerans (1), Enterobacter spp (1), S. odorifera } \\
\text { (1), Ps. aeruginosa (2), Ps. maltophila (1), Candida spp (1) }\end{array}$ \\
\hline Thigh $(\mathrm{n}=3)$ & Dirty (3) & Bacillusspp (2), P. aeruginosa (1), Bacteroides (1) \\
\hline Scrotal $(\mathrm{n}=4)$ & Dirty (4) & S. aureus (1), S. epidermidis (1), S. saprophyticus (1), K. pneumonia (1) \\
\hline $\begin{array}{l}\text { Leg/Limb } \\
(\mathrm{n}=16)\end{array}$ & Dirty (16) & $\begin{array}{l}\text { S. aureus (3), S. epidermidis (1), Bacillus spp (2), E. coli (2), P. mirabilis (1), Citrobacter spp (1), Proteus spp (2), S. } \\
\text { odorifera (3), P. aeruginosa (2), Anaerobic cocci (1), Bacteroides (2), Candida spp (3) }\end{array}$ \\
\hline Burns $(\mathrm{n}=8)$ & Contaminated (8) & $\begin{array}{l}\text { S. aureus (3), Bacillus spp (1), Enterococcus spp (1), E. coli (1), E. agglomerans (1), Enterobacter spp (1), S. odorifera } \\
\text { (1), Ps. aeruginosa (2), Ps. maltohhila (1), Candida spp (1) }\end{array}$ \\
\hline Thigh $(\mathrm{n}=3)$ & Dirty (3) & (1), Bacteroides (1) \\
\hline
\end{tabular}

Aerobic gram positive organisms accounted for $41.3 \%$ CoNS, Bacillus spp and Enterococcus spp accounted of the total number of organisms. S. aureus constituted for $18.3 \%, 8.7 \%, 11.1 \%$ and $2.4 \%$ of the total isolates $44.2 \%$ of the gram positive pathogens, coagulase nega- respectively. Aerobic gram negative organisms accounttive staphylococci (CoNS), Bacillus spp and Enterococ- ed for 42.1\% of the total isolates and Pseudomonas cus species accounted for $21.2 \%, 26.9 \%$ and $5.8 \%$ re- aeruginosa and Escherichia coli constituted $26.4 \%$ and spectively. The CoNS isolated included S. epidermidis, $24.5 \%$ of the gram negative pathogens respectively. S. saprophyticus and S. xylosus. Overall, S. aureus, The remaining aerobic gram negative isolates are of the family Enterobacteriaceae (Table 1).

TABLE 1. Bacteria and Fungal Isolates Recovered From Surgical Wound Infections

\begin{tabular}{lll}
\hline Serial No & Organism & No $(\%)$ \\
\hline 1. & Staphylococcus aureus & $23(18.2)$ \\
2 & S. epidermidis & $5(4.0)$ \\
3 & S. saprophyticus & $2(1.6)$ \\
4 & S.xylosus & $1(0.8)$ \\
5 & Staphylococcus spp & $3(2.4)$ \\
6 & Bacillus spp & $14(11.1)$ \\
7 & Enterococcus spp & $4(3.2)$ \\
8 & Escherichia coli & $13(10.3)$ \\
9 & Enterobacter agglomerans & $3(2.4)$ \\
10 & Enterobacter spp & $1(0.8)$ \\
11 & Klebsiella pneumonia & $2(1.6)$ \\
12 & Proteus mirabilis & $2(1.6)$ \\
13 & Proteus penneri & $1(0.8)$ \\
14 & Citrobacter spp & $1(0.8)$ \\
15 & Proteus spp & $2(1.6)$ \\
16 & Serratia odorifera & $6(4.7)$ \\
17 & Pseudomonas aeruginosa & $14(11.1)$ \\
18 & Ps. maltophila & $2(1.6)$ \\
19 & Pseudomonas spp & $6(4.7)$ \\
20 & Anaerobic cocci & $4(3.2)$ \\
21 & Bacteroides & $5(4.0)$ \\
22 & Gram. Positive anaerobic rods & $1(0.8)$ \\
23 & Candida spp & $11(8.7)$ \\
\hline
\end{tabular}


Pure cultures of pathogens most commonly yielded $\mathrm{S}$. The susceptibility pattern of all the bacterial strains is aureus, $22.0 \%$; Bacillus, $12.0 \%$; Ps. aeruginosa, $8.0 \%$ summarized in Table 3.

and CoNS, $8.0 \%$.

TABLE 3. Sensitivitv profile of bacterial isolates from Surgical Wound infections

\begin{tabular}{|c|c|c|c|c|c|c|c|c|c|c|c|c|}
\hline \multirow[t]{2}{*}{ Organism } & \multirow{2}{*}{$\begin{array}{l}\text { No of } \\
\text { Isolates }\end{array}$} & \multicolumn{11}{|c|}{ No of isolates resistant $(\%)$} \\
\hline & & Chl & Cip & of & Ery. & PV. & PP. & $\mathrm{CE}$ & DX & TR & FU & TM \\
\hline S. aureus & 20 & $7(35.0)$ & 0 & 0 & $14(70.0)$ & $20(100)$ & $20(100)$ & $19(95.0)$ & $18(90.0)$ & $18(90.0)$ & $9(45.0)$ & $9(45.0)$ \\
\hline CoNS & 11 & $6(54.5)$ & $1(9.1)$ & 0 & $10(90.9)$ & $11(100)$ & $11(100)$ & $11(100)$ & $11(100)$ & $11(100)$ & $9(81.8)$ & $9(81.8)$ \\
\hline Bacillus spp & 14 & $5(35.7)$ & $5(35.7)$ & $4(28.6)$ & $14(100)$ & $14(100)$ & $14(100)$ & $14(100)$ & $14(100)$ & $8(57.1)$ & $11(78.6)$ & $7(50.0)$ \\
\hline Enterococcus spp & 3 & $1(33.3)$ & 1(33.3) & 1(33.3) & $1(33.3)$ & $2(66.7)$ & $2(66.7)$ & $2(66.7)$ & $2(66.7)$ & $2(66.7)$ & $2(66.7)$ & $1(33.3)$ \\
\hline Enterobacter & 4 & $1(25.0)$ & $1(25.0)$ & $2(50.0)$ & $3(75.0)$ & $4(100)$ & $4(100)$ & $4(100)$ & $4(100)$ & $4(100)$ & $4(100)$ & $2(50.0)$ \\
\hline E. coli & 13 & $5(38.5)$ & $2(15.4)$ & $6(46.2)$ & $10(76.9)$ & $13(100)$ & $13(100)$ & $13(100)$ & $13(100)$ & $13(100)$ & $8(61.5)$ & $4(30.8)$ \\
\hline K. pneumonia & 2 & $1(50.0)$ & $1(50.0)$ & $2(100)$ & $1(50.0)$ & $2(100)$ & $2(100)$ & $2(100)$ & $2(100)$ & $2(100)$ & $2(100)$ & $1(50)$ \\
\hline Proteus spp & 5 & $4(80.0)$ & $2(40.0)$ & $4(80.0)$ & $4(80.0)$ & $5(100)$ & $5(100)$ & $5(100)$ & $4(80.0)$ & $4(80.0)$ & $4(80.0)$ & $4(80.0)$ \\
\hline Citrobacter $\mathrm{spp}$ & 1 & 0 & 0 & 0 & 0 & 0 & 0 & 0 & 0 & 0 & 0 & 0 \\
\hline Serratia odorifera & 6 & $4(66.7)$ & 2(33.3) & $4(66.7)$ & $4(66.7)$ & $6(100)$ & $6(100)$ & $6(100)$ & $6(100)$ & $4(66.7)$ & $4(66.7)$ & $3(50.0)$ \\
\hline Ps. aeruginosa & 14 & $4(28.6)$ & $5(35.7)$ & $8(57.1)$ & $10(71.4)$ & $14(100)$ & $14(100)$ & $14(100)$ & $11(78.6)$ & $8(57.1)$ & $10(71.4)$ & $4(28.6)$ \\
\hline Pseudomonas spp & 8 & $4(50.0)$ & $3(37.5)$ & $4(50.0)$ & $6(75.0)$ & $8(100)$ & $8(100)$ & $8(100)$ & $6(75.0)$ & $3(37.5)$ & $6(75.0)$ & $4(50.0)$ \\
\hline Bacteroides & 5 & $3(60.0)$ & $3(60.0)$ & $3(60.0)$ & $5(100)$ & $5(100)$ & $5(100)$ & $5(100)$ & $5(100)$ & $3(60.0)$ & $5(100)$ & $3(60.0)$ \\
\hline
\end{tabular}

Ofloxaxin (Of), chloramphenicol (Chl), cephalothin (CE), erythromycin (Ery), ciprofloxacin (Cip), penicillin V (PV), Fusidic acid (FU), tobramycin (TM), Trimethoprim (TR), cefadroxil (DX), Piperacillin (PP).

Sensitivity of the isolates to different antibiotics varied of antibiotic resistance in all pathogens isolated. Of all and most isolates were multidrug resistant. In general, the antibiotics tested, ciprofloxacin was shown to be the resistance to the $\beta$-lactam antibiotics was above $98 \%$ one most likely to be effective in treating infections as, except for cephadroxil which showed a resistance of in contrast to other antimicrobial agents tested in this erythromycin, fusidic acid and trimethoprim. Only two of the five Bacteriode spp tested was sensitive to metronidazole (result not shown).

cudy, less than $30 \%$ of the bacterial isolates were found to be resistant to its activity.

Bacterial pathogens were isolated from all the specimens while the yeast Candida species(spp) was isolated The staphylococcal pathogens were $100 \%$ sensitive to from $12.4 \%$ of them. A high prevalence of aerobic bacall the fluoroquinolones tested but the CoNS had a terial pathogen was obtained. This is in accordance to susceptibility of $89.9 \%$ to ciprofloxacin. The resistance other similar findings and confirms the importance of of S. aureus to chloramphenicol and erythromycin was aerobes in surgical wound infections ${ }^{11,17}$. In addition to $35.0 \%$ and $70.0 \%$ respectively.

\section{Discussion}

The study gives an insight to the causative pathogens of post operative wound infections in this hospital and their sensitivity profiles. It is concluded that surgical wound infections in this health institution were polymicrobic in nature and, in most cases, associated with S. aureus, Pseudomonas aeruginosa, E. coli and other pathogens. Results also showed that there is a high rate with the findings of another study reported earlier at another major teaching hospital in South Western Nigeria $^{18}$. Our study also agrees with the Nosocomial in- fection national surveillance service (NINSS) survey of (1997-2001) which reported Staphylococcus (47\%) including S. aureus and S. epidermidis as the most common organisms causing surgical site infection $\mathrm{s}^{20}$. Similar reports have been documented in an Indian Hospitals ${ }^{21}$ The prevalence of $\mathrm{S}$. aureus in surgical wound infections has been attributed to the high rate of nasal carriage of this organism in patients and health care workers involved in the treatment of the patients ${ }^{22}$. The environment of operating suite has also been incriminated as an important source of bacterial colonizing surgical wound at the centre ${ }^{23}$. This is supported by the high rate of isolation of Bacillus spp in this study. These observations suggest the need for an improved infection control programme in the centre.

The organisms causing nosocomial infections have changed in medical practice over the years ${ }^{24}$. Whereas gram positive organisms were the predominant organisms involved in these infections, gram negative organ-
isms are now being isolated at an increasing rate ${ }^{25}$. This shift may result from the greater complexity of the structure of the gram negative bacteria cell wall that made it to have intrinsic resistance to most antibacterial agents in use in the hospitals. This is shown in the high prevalence of Pseudomonas aeruginosa, and Escherichia coli, Klebsiella, Proteus, Enterobacter and other enterobacteria isolated in our study. Increasing isolation rate of Serratia odorifera as a pathogen in surgical wound infections as reported by other researchers ${ }^{11,17}$ was also observed in our study.

It has been documented that the type of organisms infecting surgical wound is a reflection of the body system involved in the surgical operation ${ }^{26}$. According to the reports, these organisms which are normal inhabitants of the body system usually become opportunistic pathogens when their niche is violated. For example, if the gastro intestinal tract is violated then E. coli and Bacteroides are common isolates ${ }^{26}$. Similarly, if urinary tract is involved, S. saprophyticus, other CoNS, Pseudomonas and Proteus are the pathogens that would be most common ${ }^{26}$. The results of this study is actually in line with this position as there was a high rate of isolation of the enterobacteria and Bacteroides from operations that involve the head, chest wall, legs and abdomen. The organisms isolated from other part of the body were also good reflections of the microflora associated with those parts e.g S. saprophyticus from the scrotal sacs.
P. aeruginosa is an epitome of opportunistic nosocopathogens which is responsible for a wide range of infections and leads to substantial morbidity and mortality ${ }^{27}$. The incidence of postoperative wound in fections due to Pseudomonas is high in this study. This corroborates the earlier studies ${ }^{10}$ and it actually calls for a need to control the increasing relaxation of general hygienic measures in the community and increasing availability and usages of low quality antimicrobials. Antibiotics were screened based on their chemical groups which reflect their modes of action, activities and mechanisms of resistance. These groups include; the $\beta$-lactams (penicillin and cephalosporin [ $\beta$-lactamase susceptible or stable]), the macrolides (e.g. erythromycin, tobramycin), the fluoroquinolones (e.g. ofloxacin, ciprofloxacin), chloramphenicol and fusidic acid. The choices depends on their availability and use at the hospital.

Ciprofloxacin has been identified as the most potent drug available for the treatment of P. aeruginosa infection $^{27}$. Our results showed that about $40 \%$ of the Pseudomonas species and $20 \%$ of the enterobacteria already demonstrated resistance to ciprofloxacin. However, in comparison with other antibiotics screened, our results showed that P. aeruginosa and other Pseudomonas spp isolated in this study demonstrated the lowest rate of resistance to ciprofloxacin. Similarly, although at a lower rate, reduced resistance of P. aeruginosa to ciprofloxacin has been reported in Jamaica in Latin America $(19.6 \%)$, in Ilorin in Nigeria $(24.7 \%)$, in India $(26.22 \%)$ and in Kualar Lumpur $(11.3 \%)^{27}$.

It is to be noted however that, these observations under score the need for urgent steps to arrest the increasing incidence of resistance to the fluoroquinolones in this environment

The results of this study indicated that Bacteroides isolates demonstrated high sensitivity to chloramphenicol, tobramycin, trimethoprim, metronidazole and the quinolones (ciprofloxacin and ofloxacin) being about $60 \%$ sensitive, whereas resistance to the $\beta$-lactam antibiotics (Penicillin V, Piperacillin, cephalothin and cephadroxil) were very high. These results are contrary to that obtained for anaerobes isolated from orofacial infections in an earlier study which reported good activities of the later agents against the anaerobes ${ }^{2}$. 
quired infections. In addition to this observation, al- excess length of stay, and extra cost. Infection Control and though peri- operative prophylaxis has been shown Hospital Epidemiology 2002; 3(4):183-189.

to decrease the incidence of wound infection ${ }^{1}$, the 7. Mangram AJ, Horan TC, Pearson ML, Silver LC, susceptibility data obtained in this study also suggested Jarvis WR. Guideline for prevention of surgical site inthat most of the antibiotics used in this study would fection, 1999. Hospital Infection Control Practices Adhave very limited usefulness for the prophylaxis or the visory Committee. Infection Control Hospital Epidemiolog empirical treatment of these infections ${ }^{3,29,30}$. Our find- 1999; 20(4):250-278.

ings support the reported increasing trends of antibi- 8. Oluwatosin OM. Surgical Wound Infection: A Genotic resistance worldwide.

A regular surveillance should be carried out to monitor the susceptibility of these pathogens and chose appropriate regimens both for prophylaxis and treatment of surgical wound infections. There is a need to develop a viable antibiotic policy and draft guidelines to prevent or reduce indiscriminate use of antibiotics, and preserve their effectiveness for better patient management.

Continuous dialogue between the microbiology department and the surgeons is strongly advised in keeping with preventing and controlling surgical wound infections at minimal cost. This will encourage rational use of antimicrobial agents and help in curbing the menace of resistance to these agents.

\section{References}

1. Bowler PG, Duerden BI, Armstrong DG. Wound Microbiology and Associated Approaches to wound management.Clinical Microbiology Review 2001; 14(2):244 -269 .

2. Nichols RL. Current Strategies for Prevention of Surgical Site Infections. Curr Infect Dis Rep 2004; 6(6):426434.

3. Surucuoglu S, Gazi H, Kurutepe S, Ozkutuk N, Ozbakkaloglu B. Bacteriology of surgical wound infection in a tertiary care hospital in Turkey. East Afr Med J 2005; $82: 331-336$

4. Tesfahunegn Z, Asrat D, Woldeamanuel Y, Estifano $\mathrm{K}$. Bacteriology of surgical site and catheter related urinary tract infections among patients admitted in Mekele Hospital,Mekele, Tigray, Ethiopia. Ethiopian Medical Journal 2009; 47:117-122.

5. Ashby E, Haddad FS, O’Donnell E, Wilson AP. How will surgical site infection be measured to ensure "high quality care for all"? The Journal of Bone and Joint Surger 2010; 92(9):1294-1299.

6. Whitehouse JD, Friedman ND, Kirkland KB, Richardson WJ, Sexton DJ. The impact of surgical-site infections following orthopedic surgery at a community hospital and a university hospital: adverse quality of life,

eral Overview. Annals of Ibadan Postgraduate Medicin 2005; 3(2):26-31

9. Lilani SP, Jangale N, Chowdhary A, Daver GB. Surgical site infection in clean and clean- contaminated cases. Indian Journal of Medical Microbiology 2005; 23:249-252 10. Emele FE, Izomoh MI, Alufolai E. Micro organisms associated with wound infections in Ekpoma, Nigeria. West Africa Journal of Medicine 1999; 18(2):97-100 11. Shinagawa N, Hirata K, Katsuramai T. et al. Bacteria isolated from surgical infections and its susceptibilities to antimicrobial agents - special references to bacteri isolated between April 2003 and March 2004. Japan Journal of Antibiotics 2005; 58(2):123 -158.

12. Cheesebrough M. District Laboratory Practice in Tropical Countries; Part 2, 2nd Edition. Cambridge; Arnold. 2006; pp 80-85.

13. Gibson PA, Khoury J. Storage and survival of bacteria by ultrafreeze. Letters in Applied Microbiology 1986, 3:127-129.

14. Barrow G, Feltham R. Cowan and Steel's Manual for the identification of medical bacteria. Cambridge University Press, London. 1993.

15. Ieven M, Verhoeven J, Pattyn SR, Goossens H. Rapid and economical method for species identification of clinically significant coagulase negative staphylococci. Clin Microbiol 1999; 33:1060-1063.

16. Clinical and Laboratory Standards Institute. M100S19- Performance Standards for Antimicrobial Susceptibility Testing; Seventeenth Informational Supplement. Clinical and Laboratory Standards Institute, Wayne, PA. 2009. 17. Giacometti A, Cirioni O, Schimizzi AM, Del Prete Epidemiology and microbiology of surgical wound in fections. J Clin Microbiol 2000; 38(2):918-922.

18. Oni AA, Bakare RA, Okesola AO, Ogunlowo NA, Ewete AF. Pattern of bacterial pathogens in surgical wound infections. African Journal of Medicine and Medical Sciences 1997; 26(3-4):139-140.

19. Shittu AO, Kolawole DO, Oyedepo EAR. Wound Infections in Two Health Institutions in Ile-Ife, Nigeria: Results of a cohort study. Ostomy Wound Management 2003; 49:52-57
24. Scheckler WE, Scheibel W, Kresge D. Temporal
20. Surveillance of surgical site infections in English hospitals 1997-2001. Nosocomial Infection National Surveillance Service (NINSS). PHLS publications, 2001.

21. Kownhar H, Shanker EM, Vignesh R, Sekar R, Velu $\mathrm{V}$, Rao VA. High rate of isolation of $\mathrm{S}$. aureus from Surgical Site Infections in an Indian Hospital. J Antimicrobial Chemotherapy 2008; 61(3):758- 760.

22. Dixon G. Sources of Surgical Infection. Surger 2002; 179-196.

Experimental Microbiology 2004; 5(1):108-118. Wer DJ, Raasch R, Rutala WA. Nosocomial infection in the ICU, the growing importance of antibiotic resistance pathogens. Chest 1999; 115(3 suppl): 34S-41S, 27. Goswami NN, Trivedi HR, Goswami AP, Patel TK, Tripathi CB. Antibiotic sensitivity profile of bacterial pathogens in postoperative wound infections at tertiar care hospital in Gujarat, India. J Pharmacol Pharmacother 2011; 2:158-164

23. Ako-nai KA, Adejuyigbe O, Adewumi TO, Lawal 28. Ndukwe KC, Okeke IN, Akinwande JA, OO. Sources of intra operative bacterial colonization Aboderin AO, Lamikanra A. Bacteriology and anof clean surgical wound infection and subsequent post timicrobial susceptibility profile of agents of orofacial operative wound infection in a Nigerian hospital. East infections in Nigerians. African Journal of Clinical and Ex African Medical Journal1992; 49(9):500-507. trends in septicemia in a community hospital. American Journal of Medicine 1991; 91(Suppl.3B):90S-94S.

25. Onipede AO, Oluyede CO, Aboderin AO, Zailani SB, Adedosu AM, Oyelese AO, Badmus TA, Ikem I, Balogun CO, Olafimihan KF. A Survey Of Hospita Acquired Infections In Obafemi Awolowo Universit Teaching Hospital, Ile-Ife. African Journal of Clinical and perimental Microbiology 2004; 5(3):272-277.

29. Adesunkanmi ARK, Badmus TA, Ogunrombi BO Audit of antibiotic therapy in acute abdomen in a $\mathrm{Ni}$ gerian Teaching Hospital. Nigerian Journal of Surgical Sciences 2004; 14:1-12.

30. Adesunkanmi ARK, Badmus TA. Pattern of aneralized peritonitis in semi-urban and rural $\mathrm{Ni}$ gerians. Chemotherapy 2006; 52(2):59-72. tibiotic therapy and clinical outcome in acute gen- 